\title{
El Mandato del Dispositivo de Registro Electrónico, reglas de las horas de servicio e Implicaciones para los Transportistas de Productos del Sudoeste de Florida ${ }^{1}$
}

\author{
Fritz Roka, Tara Wade, Luis Peña-Lévano, y Craig Sprouse ${ }^{2}$
}

El Mandato del Dispositivo de Registro Electrónico (ELD por sus siglas en inglés), se convirtió en mandato para vehículos motorizados comerciales (CMV) el 18 de diciembre del 2017 (FMSCA, 2017a). El 18 de junio del 2018, después de 90 días de extensión, el mandato también se aplicó a transportistas de productos. El propósito principal detrás del ELD fue asegurar el cumplimiento con los requerimientos de Horas de Servicio (HOS) por autotransporte y sus conductores (Omnitracs 2018a). Este artículo se enfoca en el movimiento interestatal de propiedades (productos), y sus objetivos son: 1) revisar las reglas HOS; 2) clarificar las excepciones agrícolas a las reglas HOS; y 3 ) ofrecer una discusión preliminar a cómo los ELD podrían afectar a los productores del sur de Florida.

\section{Reglas de las Horas de Servicio (HOS)}

Durante los 1930s la industria interestatal americana fue expandida rápidamente y un monto significativo de transporte comenzó a moverse en la zona continental de los Estados Unidos (Park 2018). Los recorridos de largas distancias promovieron preguntas sobre la seguridad y los expertos encontraron relación causal entre la fatiga de los conductores y los accidentes en la vía expresa. Debido a ello, las primeras reglas sobre horas de servicios para conductores de CMV fueron establecidas. Yager (2009) enfatizó la evolución de las reglas HOS desde los 1930s hasta el 2008. En 1937, un conductor podía operar un CMV hasta 10 horas durante un periodo de 24 -horas y tenía que estar "fuera de servicio" por al menos 8 horas consecutivas antes de empezar otro periodo de 24 horas. El total de "horas en servicio" era un máximo de 60 horas sobre 7 días consecutivos. Los cambios en 1962 brindaron a los conductores mayor flexibilidad para acortar los "periodos de servicio" de 24 a 15 horas, entendiendo los descansos durante el día. Un conductor tenía que estar "fuera de servicio" por al menos 8 horas consecutivas por día y el "total de servicio" fue limitado a 60 horas sobre 7 días, o 70 horas sobre 8 días. Los mayores cambios a HOS ocurrieron en el 2003 cuando los conductores podían incrementar su tiempo de manejo de 10 a 11 horas, pero solamente dentro de un más corto periodo de 14-horas de manejo, no extensible con recesos. Las horas consecutivas "fuera de servicio" incrementaron de 8 a 10 horas. Los límites semanales, 60 horas sobre 7 días, o 70 horas sobre 8 días, se mantuvieron, pero ahora los conductores podían "reiniciar" su registro de "horas de servicio" al tomar un descanso de 34 horas consecutivas. Aparte de requerir un periodo de 30-minuto de descanso después de 8 horas de manejo, las reglas HOS han permanecido sin cambios hasta septiembre del 2018 (FMCSA 2017b).

1. This document is FE1058, one of a series of the Food and Resource Economics Department, UF/IFAS Extension. Original publication date May 2019. Visit the EDIS website at https://edis.ifas.ufl.edu for the currently supported version of this publication.

2. Fritz Roka, associate professor of agricultural economics; Tara Wade, agricultural and natural resource economist, Food and Resource Economics Department; UF/IFAS Southwest Research and Education Center; and Craig Sprouse, Sunripe Certified Brands; UF/IFAS Extension Gainesville, FL 32611. Translated by Luis Peña-Lévano, lecturer; Food and Resource Economics Department, UF/IFAS Gulf Coast Research and Education Center, Plant City, FL 33563.

The Institute of Food and Agricultural Sciences (IFAS) is an Equal Opportunity Institution authorized to provide research, educational information and other services only to individuals and institutions that function with non-discrimination with respect to race, creed, color, religion, age, disability, sex, sexual orientation, marital status, national origin, political opinions or affiliations. For more information on obtaining other UF/IFAS Extension publications, contact your county's UF/IFAS Extension office. 


\section{Excepciones Agrícolas}

La agricultura recibe excepciones a las reglas de HOS siempre y cuando los transportistas de carga de "mercancías agrícolas" o "suministros granjeros para fines agrícolas" se encuentren dentro de las 150-millas aéreas (o 172.6 millas terrestres) de la "fuente agrícola". Mercancías agrícolas se refieren a cualquier cultivo, alimento no procesado, producto para consumo animal, fibra, o ganado (incluyendo peces, abejas e insectos). Suministros granjeros para fines agrícolas se definen como productos destinados para crecimiento o cosecha de commodities agrícolas durante las estaciones de plantación o cosecha. Cada estado especifica sus propias estaciones de plantación y cosecha. La fuente de una mercancía agrícola es la localización donde la mercancía es cargada a un CMV vacío(https://www.fmcsa.dot. gov/regulations/hours-service/\%E2\%80\%9Cagriculturalcommodity\%E2\%80\%9D-exception-49-cfr-3951k1hours-service-regulations). La ubicación debe ser algún almacén intermedio o lugar de manejo lejano a la finca o campo de cultivo condicionada a que la mercancía mantiene su condición y forma original y no ha sido significativamente modificada durante algún procesamiento o empaquetado. Si un conductor hace múltiples paradas durante el curso de un solo viaje, la excepción de las 150-millas aéreas se aplica solamente a la ubicación de la fuente original y termina cuando todos los productos agrícolas son descargados en el destino final del viaje. El punto de destino puede llegar a convertirse en una nueva fuente para un nuevo viaje y la excepción agrícola del radio de 150-millas aéreas seria alrededor de esa fuente.

Para un expedidor/productor de tomates, la fuente agrícola cambia dependiendo de lo que es enviado y donde ocurre el envío. Mover plantas de una casa de trasplante a los campos hace del vivero una "fuente agrícola" durante el tiempo de plantación. La fuente cambia de los campos cuando el agricultor traslade la fruta cosechada a la planta de postcosecha. Cuando las cajas de tomates empaquetadas son trasportadas a instalaciones de reempaque, o a otro almacén, o a otro punto de distribución de venta por mayor/menor, la planta de postcosecha se convierte en una "fuente agrícola". Con respecto al último escenario de movimiento de commodities entre plantas de postcosechas y otros destinos dentro de la cadena de suministros, la excepción agrícola a la regla HOS requiere que la mercancía retenga su forma original y no haya sido significativamente alterada por el procesamiento o empacado. Mientras el tomado fresco de mercado sea calificado y madurado en la planta de postcosecha, sus condiciones son fundamentalmente las mismas de cuando fue recogida del campo. Por tanto, la excepción las 150-millas-aéreas puede ser usada en una planta postcosecha de tomate. Jugos de naranja, por otro lado, pueden ser alteradas cuando son exprimidas en una planta de procesamiento. La planta de procesamiento de jugos no se consideraría una "fuente agrícola", y el transporte del jugo de naranja no calificaría para ser exenta del HOS.

La implicación importante de la excepción agrícola es que un conductor de CMV "en servicio" y sus horas de manejo mientras transporte productos agrícolas a 150 millas aéreas de su fuente no son contadas entro de los limites diarios y semanales de la HOS. Por ejemplo, considere el envío de tomates redondos desde Immokalee, FL, a Hunts Point, NY. El conductor empieza su día a las 7:00 am y dedica la primera hora para llenar combustible e inspeccionar la seguridad de su camión y remolque. A las 8:00 am comienza a cargar su remolque. Para el mediodía, su remolque está completamente lleno y comienza su viaje a Nueva York. A las 3:00 pm alcanza las cercanías del norte de Orlando, FL, lo cual enmarca el arco de las 150 millas-aéreas (172 millas-terrestres) desde Immokalee. Debido a la excepción agrícola, los cálculos particulares de la HOS comienzan de manera efectiva a las 3:00 pm. Factiblemente, el conductor podría conducir hasta las 2:00 am de la mañana siguiente antes de llegar del límite diario de HOS de las 11-horas - aunque, técnicamente, el conductor es requerido tomar un descanso obligatorio de 30-minutos cada 8 horas consecutivas de manejo. El manejo de un CMV por 14 horas consecutivas como parte de un día de 19-horas trae dudas sobre su prudencia y seguridad. Sin embargo, tal día debería ser legalmente permitido bajo la excepción agrícola.

Aunque las excepciones agrícolas permiten a los conductores de transporte de carga agrícola ser exentos a los cálculos de HOS dentro del radio de 150 millas-aéreas, los conductores y portadores de vehículos motorizados deben mantener registros de sus estatutos de actividad (RODS) por 7 días consecutivos más el día actual (FMSCA 2018). Bajo la excepción agrícola, un conductor podría marcar en su cuaderno o anotador sus ELD con "excepción agrícola" para el tiempo que estuvo conduciendo y trabajando dentro del radio de las 150 millas-aéreas.

\section{Dispositivos de Registro Electrónico (ELDs)}

Portadores de vehículos motorizados han estado experimentando con dispositivos de registro automático desde los 1980s. Los primeros dispositivos eran muy caros. En 1995 , un solo dispositivo alcanzó a costar incluso \$2500 (Omnitracs 2018b). Ya por el 2016, los avances en la 
tecnología GPS y software presionaron la reducción del costo a menos de $\$ 200$ (Cruz 2017). El acta del congreso del 2012, Movimiento Hacia el Progreso en el Siglo 21 (MAP-21), requería que todos los transportes de carga usando CMV sean equipados con ELDs. La regla final para ELD fue establecida en diciembre del 2015 y enmarcó un periodo de cuatro años consistente de tres fases para su cumplimiento. En la Fase 1, diciembre del 2015 y diciembre del 2017 fue una transición de periodo. Durante esta fase, el uso de ELD era voluntario y los operadores tenían la opción de continuar con el uso de cuadernos o utilizar los dispositivos previamente instalados. En la Fase 2, del 18 de diciembre del 2017 al 16 de diciembre del 2019, se requiere que todos los camiones tengan instalados ELDs a menos que hayan instalado previamente un sistema de registro electrónico alternativo antes de diciembre del 2017. En la Fase 3, la cual comienza a partir del 16 de diciembre del 2019, requiere que todos los conductores y portadores de vehículos motorizados usar ELD-auto certificados que sean registrados con FMSCA (FMSCA 2017a). Un ELD certificado debería:

1. conectar directamente al motor del camión y proveer registro continuo mientras el CMV está operando;

2. permitir que el conductor seleccione una de las tres opciones - "en servicio, manejando", "en servicio, no manejando", "fuera de servicio";

3. rápidamente proveer leyes de aplicación con una muestra grafica del registro del conductor de horas de servicio; $y$

4. transferir datos vía sistema inalámbrico o con un portador USB.

ELDs pueden acomodar la excepción agrícola a las reglas HOS. Dentro del radio de las 150 millas-aéreas de una "fuente agrícola", un conductor CMV puede anotar en su ELD que está operando bajo "excepción agrícola”. Existen algunas excepciones para la norma ELD el cual aplica para comercio interestatal:

1. Todos los CMVs que son modelos mas viejos que el año 2000 son exentos de usar ELS;

2. conductores, agricultores u otros, que no operan fuera del limite HOS por mas de 8 de 30 días, son exentos de comprar o mantener ELDs.

En ninguna circunstancia los ELDs cambian las reglas HOS. El objetivo principal para ordenar ELDs es asegurar que portadores de vehículos motorizados y sus conductores cumplan con las reglas actuales de HOS (Omnitracs 2018). Los cuadernos de anotaciones no son vistos como registro confiable de lo que los conductores realmente acumulan como horas en servicio y horas de manejo consecutivas. Defensores de los ELDs argumentan que los ELDs incrementarán la exactitud de los reportes de HOS, y reducirán el papeleo necesitado para el cumplimiento de las HOS, e incrementarán la eficiencia de programación para despachadores al mejorar la comunicación entre conductores y despachadores (Omnitracs 2018b). ELDs también tienen la capacidad de grabar incidencias de velocidad, frenados bruscos y otras funciones del motor. Registros precisos de las operaciones del CMV pueden también proteger a los conductores de expectativas irrealistas o inseguras del empleador que puede promover exceso de velocidad o violaciones contra las reglas HOS.

\section{Implicaciones de los ELDs para los productores del sur de la Florida}

FMSCA estimó que la reducción del costo de papeleo e incremento en la eficiencia del uso de combustibles como resultado de la adopción de ELDs podría reducir alrededor $\$ 1.6$ billones anuales en costos de carga en todo Estados Unidos (Omnitracs 2018a). Muchos portadores de vehículos han argumentado que el costo de instalación de la nueva tecnología ELD y el cumplimiento mas estricto con las reglas HOS podría contrarrestar estas ganancias (Cruz 2017). Los costos de transporte en camiones han incrementado significativamente. Datos de abril del 2018 muestran que estas tasas son mayores al 20\% de lo que fueron en marzo del 2017 (Karst 2018a). Recientemente en junio del 2018 , las tasas han incrementado entre 16 y $25 \%$ comparados al mes anterior (Karst 2018b). El alcance por el cual los ELDs son en parte responsables por el incremento de estas tasas es incierto. El fuerte crecimiento de la economía de los Estados Unidos, bajo nivel de desempleo, y escasez de conductores CDL calificados son importantes razones del reciente crecimiento de estas tasas. El costo de instalación de ELDs podría forzar a más pequeños e independientes operadores propietarios a salirse de la industria de carga. $\mathrm{Si}$ se da este caso, entonces la falta de CMVs y conductores podría exacerbar y posiblemente incrementar incluso más las tasas de transporte y carga.

ELDs podrían afectar las tasas de carga al reforzar el cumplimiento con las reglas HOS y por tanto incrementar el tiempo total para mover productos de un punto a otro. Para ilustrar este punto, consideremos el extremo caso de cumplimiento versus no cumplimiento con las reglas HOS cuando dos (2) remolques cargados de tomates desde 
Immokalee, FL a Hunts Point, NY. Cada remolque refrigerado carga 1,700 cartones de 25-libras (CH Robinson 2017). Ahora considere ambos conductores ganando el mismo salario anual, $\$ 80,000$ al año, y trabajando/conduciendo 250 días al año, o $\$ 320$ por día. El primer conductor opera bajo las reglas HOS (cumple), mientras que el segundo conductor no cumple. Las reglas HOS restringen al primer conductor a manejar 11 horas por día, y si promedia 60 millas por hora, el viaja 660 millas por día. El segundo conductor promedia similar velocidad (i.e., $60 \mathrm{mi} / \mathrm{h}$ ), pero conduce 15 horas, cuatro horas más que el límite de HOS. El viaje de FL a NY es 1,253 millas (ttp://www.distance-cities.com). El segundo conductor maneja 900 millas por día completando su viaje en 1.4 días (1,253 millas divididas en $900 \mathrm{mi} /$ día). Cumpliendo estrictamente con las reglas HOS, el primer conductor hace el mismo viaje en 1.9 días (1,253 millas divididas en $660 \mathrm{mi} /$ día), o $36 \%$ mas tiempo que el segundo (desobediente) conductor. Asumiendo que los conductores continúan ganando el mismo ingreso diario (\$320), la diferencia en costo del primer conductor (cumplidor, \$608), y el segundo conductor (desobediente, \$448) es de \$160. Dado 1,700 cartones por carga, cumpliendo con las reglas HOS incrementó los costos al agregar tiempo de manejo de $\$ 0.09$ por cartón ( $\$ 160 / 1,700$ cartones).

Mientras que los costos de transportar tomates y otros productos afuera del sur de Florida posiblemente incrementarán al usar ELDs, una pregunta mas importante es saber cómo los ELDs afectarán los costos de carga desde otras áreas del país. Productor de México vienen de McAllen, TX y Nogales, AZ. Muchos de estos productos son enviados a mercados de la Costa del Este los cuales compiten directamente con productores del sur de la Florida. Examinamos como ELDs podrían beneficiar a los agricultores del sur de la Florida usando los siguientes parámetros:

- Destino: Hunts Point, NY;

- Capacidad del remolque: 1,700 cartones de tomates redondos verdes de 25-libras.

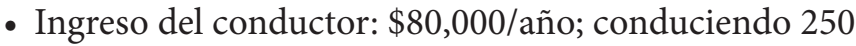
días al año; o \$320/día.

- Velocidad del manejo: 60 millas por hora.

- HOS con ELD: 11 horas por día, 600 mi/día.

- HOS sin ELD: 15 horas por día, 900 mi/día.

La tabla 1 resume el numero de días y costos promedios requeridos para completar viajes desde cada punto de inicio a Hunts Point, NY, dado los parámetros especificados. Los cortos de carga para los conductores que cumplen con las reglas HOS desde McAllen, TX y Nogales, AZ serian de 5 y 10 centavos más por cartón, respectivamente, comparado con similares conductores desde el sur de la Florida.

Mientras que los productores no pagan costos de carga para mover productos desde sus plantas postcosechas a los puntos de venta terminales, más estricta fidelidad a las reglas HOS provee a los productores del sur de la Florida con una ventaja competitiva al tener relativamente costos más bajos de transporte y carga.

\section{Comentarios de Conclusión}

ELDs son mandatos impuestos a una larga porción de la industria de carga, incluyendo camiones de transporte de producto fresco. El objetivo principal de los ELDs es asegurar que portadores de vehículos motorizados y sus conductores cumplan con las reglas actuales de HOS. Las excepciones agriculturas proveen a los productores agrícolas y los transportistas un radio de 150-millas aéreas de la "fuente agrícola" en la cual HOS ni ELDs son requeridos. Por último, los productores del sur de la Florida serán impactados por los ELDs ya que la mayoría de sus productos son exportados fuera del estado a los mercados de las Costas del Este y norte de los Estados Unidos, más allá del radio de las 150-millas aéreas. Existe aún debate si el efecto neto de las ELDs incrementará o reducirá los costos. A la larga, la adopción de la nueva tecnología por los portadores de vehículos motorizados y conductores, veremos si el costo de reducción en papeleo y/o eficiencia en uso de combustible y logística compensara el costo del equipo ELD y la flexibilidad que los conductores gozaron cuando HOS no eran registrados por las ELDs. Para los productores del sur de Florida, específicamente, un mayor cumplimiento con las reglas HOS podrían promover una ventaja competitiva porque sus productos se mercadean en las Costas del Este de los Estados Unidos, las cuales geográficamente están mas cerca que Texas o Arizona, donde la mayoría de la producción mexicana es enviada al entrar los Estados Unidos.

\section{Referencias}

Cruz, A. 2017. "ELD mandate will increase costs." American Trucker, July 31, 2017. http://www.trucker.com/print/3091. Accessed April 24, 2018.

FMCSA (Federal Motor Carrier Safety Administration). 2018. Agricultural Exceptions and Exemptions to the FMCSA Hours of Service (HOS) and Commercial Driver's 
License (CDL) Rules. June 19, 2018. http://www.fmcsa.dot. gov/elds/agricultural-exceptions-and-exemptions-fmcsasafety. Accessed June 22, 2018.

FMSCA. 2017a. Implementation Timeline. August 31, 2017. https://www.fmcsa.dot.gov/hours-service/elds/ implementation-timeline. Accessed June 2, 2018.

FMCSA. 2017b. Summary of Hours of Service Regulations. March 9, 2017. http:/www.fmcsa.dot.gov/regulations/ hours-service/summary-hours-service-regulations. Accessed June 18, 2018.

Grower Alliance. 2011. Website accessed July 2, 2018. http://groweralliance.net/products/tomatoes/

Karst, T. 2018a. "Survey shows muted supply effect of ELD enforcement." The Packer April 23, 2018. https://www. thepacker.com/article/survey-reveals-muted-supply-effecteld-enforcement. Accessed June 1, 2018.

Karst, T. 2018b. "Truck rules help produce haulers with hours of service, ELDs." The Packer. June 7, 2018. https:// www.thepacker.com/article/truck-rules-help-producehaulers-with-hours-of-service-elds . Accessed June 18, 2018.

Omnitracs. 2018a. ELD Facts. https://eldfacts.com/eldfacts/. Accessed April 18, 2018.

Omnitracs. 2018b. ELDs - Separating Facts from Fiction. https://eldfacts.com/eld-myths/. Accessed April 24, 2018.

Park, J. 2018. "Where did the Hours of Service Rules come from, anyway?” HDT Truckinginfo. March 15, 2018. https://www.truckinginfo.com/279731/where-did-thehours-of-service-rules-come-from-anyway. Accessed June $25,2018$.

Robinson, C. H. 2017. Contract Trailer Equipment. https:// www.chrobinson.com/en-us/-/media/chrobinson/newspdf/truckloadequipmentguide.pdf. Accessed July 2, 2018.

Yager, T. 2009. Commercial Motor Vehicle Drivers' Hours of Service: Background Information. Federal Motor Carrier Safety Administration, MCSAC presentation, December 7, 2009. https://www.slideserve.com/Jims/ commercial-motor-vehicle-drivers-hours-of-service 
Table 1. Costo adicional de transporte de Tomate de Hunts Point, NY, por conductores cumplidores a la regla HOS.

\begin{tabular}{|c|c|c|c|c|c|c|}
\hline \multirow[t]{2}{*}{ Punto de inicio } & \multirow[t]{2}{*}{ Millas } & \multicolumn{2}{|c|}{ Días para completar el viaje } & \multirow{2}{*}{$\begin{array}{c}\text { ELD Tiempo } \\
\text { Extra } \\
\text { Días }\end{array}$} & \multirow{2}{*}{$\begin{array}{c}\text { Costo agregado } \\
\text { en el ingreso del } \\
\text { conductor } \\
\text { \$/viaje }\end{array}$} & \multirow{2}{*}{$\begin{array}{c}\text { Costo agregado } \\
\text { por cartón } \\
\text { \$/cartón }\end{array}$} \\
\hline & & $\begin{array}{c}\text { Cumpliendo con } \\
\text { ELD }\end{array}$ & $\begin{array}{l}\text { No Cumpliendo } \\
\text { con ELD }\end{array}$ & & & \\
\hline Immokalee, FL & 1,253 & 1.4 & 1.9 & 0.5 & $\$ 160$ & $\$ 0.09$ \\
\hline McAllen, TX & 2,003 & 2.2 & 3.0 & 0.8 & $\$ 256$ & $\$ 0.15$ \\
\hline Nogales, AZ & 2,497 & 2.8 & 3.8 & 1.0 & $\$ 320$ & $\$ 0.19$ \\
\hline
\end{tabular}

